\title{
Decreased IL-1ra and NCAM-1/CD56 Serum Levels in Unmedicated Patients with Schizophrenia Before and After Antipsychotic Treatment
}

\author{
Che-Sheng $\mathrm{Chu}^{1,2 *}$, Dian-Jeng $\mathrm{Li}^{3 *}$, Chin-Liang $\mathrm{Chu}^{4}$, Chih-Ching $\mathrm{Wu}^{5,6}$, and $\mathrm{Ti} \mathrm{Lu}^{1 凶}$ \\ 'Department of Psychiatry, Kaohsiung Veterans General Hospital, Kaohsiung, Taiwan \\ ${ }^{2}$ Center for Geriatric and Gerontology, Kaohsiung Veterans General Hospital, Kaohsiung, Taiwan \\ ${ }^{3}$ Department of Addiction Science, Kaohsiung Municipal Kai-Syuan Psychiatric Hospital, Kaohsiung, Taiwan \\ ${ }^{4}$ Rong Xin Mental Health Clinic, Kaohsiung, Taiwan \\ ${ }^{5}$ Department of Medical Biotechnology and Laboratory Science, College of Medicine, Chang Gung University, Taoyuan, Taiwan \\ ${ }^{6}$ Department of Otolaryngology-Head \& Neck Surgery, Chang Gung Memorial Hospital, Taoyuan, Taiwan
}

\begin{abstract}
Objective Schizophrenia (SZ) has been associated with the inflammatory-related and immunological pathogenesis. This study investigates the aberration of cytokines in patients with $\mathrm{SZ}$.

Methods Thirty patients with SZ without antipsychotic treatment for at least two weeks participated. We measured the serum levels of fourteen cytokines at hospital admission and after 8-week antipsychotic treatment. Severity was measured by expanded version of 24 -items brief psychiatric rating scale (BPRS-E). Repeated measure analyses of variance were conducted.

Results The interleukin-1 receptor antagonist (IL-1ra) was significantly decreased after 8-week antipsychotic treatment than those of before antipsychotic treatment $(\mathrm{F}=12.15, \mathrm{df}=1 / 30, \mathrm{p}=0.002)$. Neural cell adhesion molecule $1 / \mathrm{CD} 56$ (NCAM-1/CD56) was significantly decreased $(\mathrm{F}=6.61, \mathrm{df}=1 / 30, \mathrm{p}=0.016)$ among those with second-generation antipsychotics but not first-generation antipsychotics treatment. The changes of BPRS-E-manic and BPRS-E-anxiety scores correlated with the baseline IL-1ra ( $\mathrm{r}=-0.393), \mathrm{IL}-6$ ( $\mathrm{r}=-0.407)$, and insulin like growth factor binding protein $3(\mathrm{r}=-0.446)$. Additionally, the changes of BPRS-E and BPRS-E-negative scores correlated with the changes of brain-derived neurotrophic factor $(\mathrm{r}=0.372)$ and interferon-gamma $(\mathrm{r}=0.375)$.
\end{abstract}

Conclusion Our study supports that IL-1ra and NCAM-1/CD56 may be considered as markers of developing SZ.

Psychiatry Investig 2018;15(7):727-732

Key Words Antipsychotic, Cytokines, Schizophrenia.

\section{INTRODUCTION}

Schizophrenia (SZ) is a severe and chronic psychiatric disorder that affects approximately $0.30 \%$ to $0.66 \%$ cases per 10,000 person-years. ${ }^{1} \mathrm{SZ}$ results in great patient suffering and

Received: September 20, 2017 Accepted: November 10, 2017

$\triangle$ Correspondence: Che-Sheng Chu, MD

Department of Psychiatry, Kaohsiung Veterans General Hospital, No. 386, TaChung 1st Road, Kaohsiung 81362, Taiwan

Tel: +886-7-3422121, Fax: +886-7-3468346, E-mail: youngtzuchi@hotmail.com

$\triangle$ Correspondence: Ti Lu, MD, MSc

Department of Psychiatry, Kaohsiung Veterans General Hospital, No. 386, TaChung 1st Road, Kaohsiung 81362, Taiwan

Tel: +886-7-3422121, Fax: +886-7-3468346, E-mail: tlu@vghks.gov.tw

*These authors contributed equally to this work.

(c) This is an Open Access article distributed under the terms of the Creative Commons Attribution Non-Commercial License (http://creativecommons.org/licenses/by$\mathrm{nc} / 4.0$ ) which permits unrestricted non-commercial use, distribution, and reproduction in any medium, provided the original work is properly cited. socio-economic burden, ${ }^{2}$ and it is one of the world's top 10 causes of long-term disability within the categories of sociooccupational functioning and self-care. ${ }^{3}$ Although researchers have worked diligently to find treatment strategies, $\mathrm{SZ}$ continues to be one of the most debilitating psychotic disorders due to the lack of effective treatments. ${ }^{3}$ This may be due to our poor understanding of the etiology of SZ. In addition to well-established findings of some mechanisms, such as dopaminergic and glutamatergic pathways, ${ }^{4}$ that contribute to the development of SZ, studies have indicated that the alternation of the immune-inflammatory cytokines that are produced by Th1 and Th2 cells might play a role. ${ }^{5}$ However, the findings of these studies have been inconsistent. A systemic review reported that patients with SZ have increased serum levels of the interleukin (IL)-1 receptor antagonist (IL-1ra), soluble IL-2 receptor (sIL-2R), and IL-6 and decreased levels of IL-2.5 A 
meta-analysis showed that drug-naïve patients experiencing their first episode of psychosis have significantly increased levels of pro-inflammatory cytokines, such as IL-1 $\beta$, sIL-2R, IL-6, and tumor necrosis factor (TNF)- $\alpha$, and non-significant effect size estimates were obtained for IL-2, IL-4, and interferon (IFN)- $\gamma^{6}$

In addition to inflammatory cytokines, researchers have become interested in neurotrophic factors. Because SZ is considered a neurodevelopmental disorder, ${ }^{7}$ several growth factors have recently been investigated in SZ-related research, and increasing evidence suggests that they are involved in the pathogenesis of SZ. Brain-derived neurotrophic factor (BDNF), which is one of the most abundant factors in the growth factor family, is involved in neurodevelopment, neuronal survival and differentiation, and synaptic plasticity. Although several studies have examined BDNF levels in patients with SZ, the findings have been contradictory. The severity of psychosis in patients with SZ has been reported to be negatively correlated with BDNF levels. ${ }^{8}$ However, this finding was not replicated in a subsequent study. ${ }^{9}$ Glial cell-derived neurotrophic factor (GDNF), which is a member of the transforming growth factor (TGF) $-\beta$ family, is a potent neurotrophic factor for dopaminergic neurons. ${ }^{10}$ After antipsychotic treatment, GDNF levels have been shown to increase while psychotic symptoms decrease in patients with SZ. ${ }^{10}$ However, another study demonstrated that the serum levels of GDNF did not differ between patients with SZ and healthy controls. ${ }^{11}$ Other studies have investigated the interactions between SZ and other growth factors, such as nerve growth factor $(\mathrm{NGF})^{12}$ and insulin-like growth factor (IGF-1). ${ }^{13}$ However, the involvement of growth factors in SZ is poorly understood and inconclusive.

To date, $\mathrm{SZ}$ is a disorder that is clinically diagnosed using the Diagnostic and Statistical Manual of Mental Disorder, 5th edition (DSM-5) ${ }^{14}$ Precise biomarkers are helpful for clinicians for differential diagnoses and for researchers to for the development of newer psychotropic agents. The results of the studies discussed above suggest that the association of changes in cytokines and the severity of psychosis in patients with SZ are still controversial. Furthermore, few studies have performed repeated measurements of multiple cytokines between preand post-treatment in subjects with SZ. Given these aforementioned gaps within the literature, we conducted a prospective study to investigate the relationship between cytokine levels and the severity of SZ. We aimed to assess a variety of cytokines before and after treatment and compare them in multiple groups in a naturalistic setting without a control group.

\section{METHODS}

\section{Participants}

Thirty patients from a medical center in Southern Taiwan who were admitted from August 2014 to August 2015 were enrolled in the present study. All patients met the diagnostic criteria for SZ according to DSM-5. ${ }^{14}$ The patients were unmedicated for a minimum of two weeks before enrollment. We excluded patients 1) with mental retardation, neurocognitive disorders, major depressive disorder, bipolar I or II disorder, or substance dependence or 2) who could not provide informed consent. All patients were admitted to the acute psychiatric ward and followed and treated for eight weeks with antipsychotics. This study was approved by the Ethics Committee of Kaohsiung Veterans General Hospital (VGHKS13-CT5-08).

\section{Instruments and pharmachotherapy}

The Brief Psychiatric Rating Scale (BPRS), which is one of the most frequently used screening tools, is used to quickly assess psychopathology in various psychiatric disorders. The original BPRS, which consists of 16 item, was developed in 1962. ${ }^{15}$ It was later extended to 18 items. More recently, the expanded 24-items BPRS (BPRS-E) was developed to increase the instrument's sensitivity to psychotic and affective disorders. ${ }^{16}$ The BPRS-E was administered to 30 patients before and after the eight weeks of treatment with antipsychotics, which was supervised by a psychiatrist (CLL). ${ }^{16}$ Four conceptual domains (manic excitement/disorganization, depression/anxiety, negative symptoms, and positive symptoms) were considered in a subanalysis in the present study. ${ }^{17}$ The presence and severity of psychiatric symptoms were rated from 1 (not present) to 7 (extremely severe). The total scores varied from 24 to 168 , with higher scores denoting more severe psychopathology.

During the eight-week follow-up period, the classification and dosage of the antipsychotic agents were flexibly adjusted according to the clinical judgment of the treating psychiatrists. Drug adherence was monitored and ensured by psychiatric nurses.

\section{Bioassay}

Venous blood $(5 \mathrm{~mL})$ was collected between 7:00 and 8:00 AM to avoid circadian fluctuations of the measured cytokines. After centrifugation at 3,000 rpm for $10 \mathrm{~min}$, the serum was separated from the blood and stored at $-70^{\circ} \mathrm{C}$ prior to the assay. The serum levels of the following 14 cytokines were examined in the present study: IFN- $\gamma$, IL-1 $\beta$, IL-1ra, IL-2, IL6, IL-8, IL-10, IGF binding protein 1 (IGFBP-1), IGFBP-2, IGFBP-3, BDNF, GDNF, NGF- $\beta$, and neural cell adhesion molecule 1/CD56 (NCAM1/CD56). These cytokines were 
measured using sandwich enzyme-linked immunosorbent assay kits (R\&D Systems, Inc., Minneapolis, MN, USA) according to the manufacturer's protocol. All experiments were performed in duplicate. The intra- and inter-assay variations were less than $10 \%$. The blood of all patients was assayed at admission baseline (week 0) and after antipsychotic treatment (week 8).

\section{Statistical analysis}

The data for the patient demographics and clinical characteristics are presented as mean \pm standard deviation for continuous variables and percentages for categorical variables. The primary analysis examined the effects of the eight weeks of antipsychotic treatment on the cytokines levels. Additionally, because second-generation antipsychotics (SGAs) have different binding profiles and mechanisms of action compared with first-generation antipsychotics (FGAs), we examined if the effects of SGAs differed from those of FGAs on cytokine levels. ${ }^{8}$ Therefore, we used a repeated-measure analysis of variance (ANOVA) to examine the effects of effects of treatment (time) and time $\times$ the different classes of antipsychotics (FGAs vs. SGAs) as the within-subject contrast. The secondary analyses examined the correlations between the cytokine levels and the BPRS-E scores with Spearman correlation (e.g., between baseline cytokine levels and the mean changes of cytokines and the BPRS-E-total and BPRS-E-subscales scores before and after antipsychotic treatment). All statistical analyses were conducted using SPSS 19.0 (IBM Corp., Armonk, NY, USA) and differences were considered statistically significant when p values were less than 0.05 .

\section{RESULTS}

\section{Characteristics of the sample}

Table 1 lists the sociodemographic and clinical data of the unmedicated patients with SZ. The subjects consisted of 17 men $(56.7 \%)$ and 13 women (43.3\%). All patients were unmedicated for at least two weeks before enrollment. Table 1 presents the data on age, age of illness onset, duration of illness, gender, education, body mass index, smoking, and class of antipsychotics (FGAs vs. SGAs). The mean age, age at illness onset, disease duration of the recruited patients, and mean chlorpromazine equivalent dose $(\mathrm{mg} /$ day) were $47.3 \pm 8.7$, $25.9 \pm 8.9,21.5 \pm 9.6$ years old, and $490.1 \pm 225.8 \mathrm{mg} /$ day, respectively. In addition, 21 out of the 30 patients (70\%) were treated with SGAs.

\section{Effects of antipsychotic treatment on the psychopathology and serum cytokine levels}

Table 2 shows the comparisons of the pre- and post-treat-
Table 1. Clinical characteristics of patients with schizophrenia $(\mathrm{N}=30)$

\begin{tabular}{lc}
\hline \multicolumn{1}{c}{ Variables } & Mean (SD) \\
\hline Age & $47.3(8.7)$ \\
Gender (M/F) & $17 / 13$ \\
Age of onset & $25.9(8.9)$ \\
Duration of illness (years) & $21.5(9.6)$ \\
Education & $10.13(3.501)$ \\
BMI & $26.25(5.835)$ \\
Smokers (Y/N) & $4 / 26$ \\
FGAs/SGAs & $9 / 21$ \\
Chlorpromazine equivalent dose & $490.1(225.8)$ \\
(mg/day) during study & \\
\hline Data are presented as man (SD). BMI: body
\end{tabular}

Data are presented as mean (SD). BMI: body mass index, FGAs: first-general antipsychotics, SGAs: second-general antipsychotics, SD: standard deviation

ment psychopathology and cytokine data with the factor of time $\times$ antipsychotic class interaction, which were analyzed with repeated-measures ANOVA. Eight weeks of antipsychotic treatment resulted in significant decreases in the BPRSE-total, BPRS-E-manic, BPRS-E-anxiety, BPRS-E-negative, and BPRS-E-positive subscales and Clinical Global Impression-Severity (CGI-S). The levels of only one (IL-1ra) of $14 \mathrm{cy}-$ tokines decreased significantly after antipsychotic treatment compared with before antipsychotic treatment $(\mathrm{F}=12.15, \mathrm{df}=1$ / 30, $\mathrm{p}=0.002$ ). Additionally, a repeated-measures ANOVA showed significant interactions between time $\times$ antipsychotic class for the BPRS-E-anxiety $(\mathrm{F}=7.04, \mathrm{df}=1 / 30, \mathrm{p}=0.013)$ and BPRS-E-positive $(\mathrm{F}=4.63, \mathrm{df}=1 / 30, \mathrm{p}=0.040)$ subscales and NCAM-1/CD56 ( $\mathrm{F}=6.61, \mathrm{df}=1 / 30, \mathrm{p}=0.016)$, with a trend towards an interactions for IL-1ra $(F=3.93, \mathrm{df}=1 / 30, \mathrm{p}=0.057)$.

\section{Correlation of the baseline and cytokine level changes and BPRS-E scores after eight weeks of antipsychotic treatment}

Table 3 shows that the changes in the BPRS-E-manic and BPRS-E-anxiety scores after treatment correlated with the baseline levels of IL-1ra ( $r=-0.393, p=0.032)$, IL-6 ( $r=-0.407$, $\mathrm{p}=0.025)$, and IGFBP3 ( $\mathrm{r}=-0.446, \mathrm{p}=0.014)$. Additionally, the changes in the BPRS-E and BPRS-E-negative scores correlated with the changes in the levels of BDNF $(r=0.372, p=0.043)$ and IFN $-\gamma(\mathrm{r}=0.375, \mathrm{p}=0.041)$, respectively. For the baseline psychopathology and changes in the levels of serum cytokines after eight weeks of treatment, the baseline BPRS scores correlated with the changes in the levels of IFN- $\gamma(\mathrm{r}=-0.424, \mathrm{p}=$ $0.02)$, IL-2 ( $r=-0.428, \mathrm{p}=0.018)$, and BDNF $(\mathrm{r}=-0.602, \mathrm{p}<0.001)$ (data not shown). 


\section{DISCUSSION}

Although several studies have reported an association between cytokine levels and SZ, our study compared the levels of multiple cytokines in patients with SZ before and after antipsychotic treatment. Together with the BPRS-E assessment of severity, we examined cytokines among the multiple domains of inflammatory factors, neurotrophic factors, growth factors, and immunoacting factors. One of the main findings in our study was that IL-1ra levels were significantly decreased after the eight-week antipsychotic treatment, which was sim- ilar to previous report. ${ }^{18} \mathrm{IL}-1 \mathrm{ra}$, which is a member of the IL-1 family and which is secreted by immune cells, acts as an inhibitor of the pro-inflammatory effects of IL-1 $\beta .{ }^{19}$ Cytokine actions can also be inhibited by naturally occurring cytokine receptor antagonists, such as the IL-1ra, which competes with the physiologic ligand to bind to membrane IL-1 receptors. IL1ra is produced in response to several inflammatory stimuli, including IL-1 and IL-6, and its levels increase in a variety of infections and inflammatory diseases. ${ }^{19}$ Another study has indicated that chronic low-grade systemic inflammation may lead to a two- to three-fold increase in the systemic concentra-

Table 2. Effects of antipsychotic treatment on the psychopathology and serum cytokine levels

\begin{tabular}{|c|c|c|c|c|c|c|}
\hline & \multirow{2}{*}{ Pre-treatment } & \multirow{2}{*}{ Post-treatment } & \multicolumn{2}{|c|}{ Time } & \multicolumn{2}{|c|}{ Time $\times$ antipsychotic } \\
\hline & & & $\mathrm{F}, \mathrm{df}$ & $\mathrm{p}$ value & $\mathrm{F}, \mathrm{df}$ & p value \\
\hline BPRS-E-total & $66.00(11.24)$ & $46.73(7.31)$ & $109.27,1$ & $<0.001^{*}$ & $<0.001,1$ & 0.986 \\
\hline BPRS-E-manic & $20.9(7.12)$ & $13.43(3.22)$ & $28.23,1$ & $<0.001^{*}$ & $0.01,1$ & 0.920 \\
\hline BPRS-E-anxiety & $13.37(4.74)$ & $9.53(2.30)$ & $19.39,1$ & $<0.001^{*}$ & $7.04,1$ & $0.013^{*}$ \\
\hline BPRS-E-negative & $12.67(4.09)$ & $11.17(2.56)$ & $12.85,1$ & $0.001^{*}$ & $1.87,1$ & 0.183 \\
\hline BPRS-E-positive & $19.07(3.12)$ & $12.60(2.40)$ & $211.68,1$ & $<0.001^{*}$ & $4.63,1$ & $0.040^{*}$ \\
\hline CGI-S & $4.97(1.13)$ & $3.60(0.86)$ & $50.62,1$ & $<0.001^{*}$ & $0.08,1$ & 0.778 \\
\hline IFN- $\gamma$ & $0.57(1.02)$ & $0.40(0.43)$ & $0.64,1$ & 0.431 & $0.02,1$ & 0.892 \\
\hline $\mathrm{IL}-1 \beta$ & $0.22(0.21)$ & $0.21(0.21)$ & $0.13,1$ & 0.772 & $0.01,1$ & 0.918 \\
\hline IL-1ra & $0.95(0.61)$ & $0.70(0.31)$ & $12.15,1$ & $0.002^{*}$ & $3.93,1$ & 0.057 \\
\hline IL-2 & $4.26(21.44)$ & $0.28(0.37)$ & $0.46,1$ & 0.501 & $0.39,1$ & 0.539 \\
\hline IL-6 & $1.73(2.13)$ & $1.36(1.94)$ & $0.58,1$ & 0.452 & $0.43,1$ & 0.517 \\
\hline IL-8 & $5.34(8.84)$ & $3.60(1.49)$ & $0.70,1$ & 0.411 & $0.15,1$ & 0.702 \\
\hline IL-10 & $3.26(10.23)$ & $0.92(0.89)$ & $0.98,1$ & 0.330 & $0.12,1$ & 0.736 \\
\hline IGFBP1 & $9.47(11.45)$ & $10.47(14.55)$ & $0.19,1$ & 0.890 & $0.24,1$ & 0.627 \\
\hline IGFBP2 & $122.96(62.26)$ & $119.59(68.53)$ & $0.14,1$ & 0.711 & $0.02,1$ & 0.882 \\
\hline IGFBP3 & $2.32(0.67)$ & $2.29(0.64)$ & $0.15,1$ & 0.703 & $0.06,1$ & 0.813 \\
\hline BDNF & $1.29(2.31)$ & $0.76(1.64)$ & $2.00,1$ & 0.169 & $1.76,1$ & 0.195 \\
\hline GDNF & $0.019(0.046)$ & $0.017(0.033)$ & $0.469,1$ & 0.499 & $0.469,1$ & 0.499 \\
\hline Beta-NGF & $0.023(0.095)$ & $0.018(0.068)$ & $0.365,1$ & 0.550 & $0.365,1$ & 0.550 \\
\hline NCAM-1/CD56 & $0.266(0.113)$ & $0.260(0.090)$ & $0.063,1$ & 0.804 & $6.610,1$ & $0.016^{*}$ \\
\hline
\end{tabular}

*significant differences. BDNF: brain-derived neurotrophic factor, BPRS-E: the expanded 24-items BPRS, CGI-S: clinical global impressionseverity, GDNF: glial-derived neurotrophic factor, IFN: interferon, IGFBP: insulin-like growth factor-binding protein, IL: interleukin, NCAM: neural cell adhesion molecule, NGF: nerve growth factor

Table 3. Correlation of the baseline and cytokine level changes and BPRS-E scores after eight weeks of antipsychotic treatment

\begin{tabular}{|c|c|c|c|c|c|}
\hline & IL-1ra, T0 & IL-6, T0 & IGFBP3, T0 & $\triangle \mathrm{IFN}$ & $\triangle \mathrm{BDNF}$ \\
\hline$\triangle B P R S$-total & -0.333 & -0.350 & -0.315 & 0.335 & $0.372^{*}$ \\
\hline$\triangle \mathrm{BPRS}-\mathrm{manic}$ & $-0.393^{*}$ & $-0.407^{*}$ & -0.118 & 0.322 & 0.263 \\
\hline$\triangle$ BPRS-anxiety & 0.128 & -0.229 & $-0.446^{*}$ & -0.056 & 0.283 \\
\hline$\triangle B P R S$-negative & -0.010 & -0.028 & 0.024 & $0.375^{*}$ & 0.184 \\
\hline$\triangle B P R S$-positive & -0.276 & -0.058 & -0.015 & 0.142 & -0.086 \\
\hline
\end{tabular}

${ }^{*} \mathrm{p}<0.05$, T0: data at admission, $\triangle$ : change psychopathology and serum cytokines of before and after antipsychotic treatment. BDNF: brainderived neurotrophic factor, BPRS-E: the expanded 24-items BPRS, IFN: interferon, IGFBP: insulin-like growth factor-binding protein, IL: interleukin 
tions of variable cytokines, including IL-1ra. ${ }^{20}$ Our IL-1ra results support the involvement of an immune-inflammatory mechanism in SZ. ${ }^{5}$ However, the results for the other inflammatory factors, including IFN- $\gamma$, IL- $1 \beta$, IL-2, IL-6, IL-8, and IL-10, indicated an insignificant trend of a decrease after treatment. Although several studies have explored the relationship between psychosis severity and changes in inflammatory factors, the results are inconclusive. A 28-day aripiprazole trial of chronic patients with $\mathrm{SZ}$ has shown a significant reduction in the levels of IL-1 $\beta$, IL-6, TNF- $\alpha$, IL-1ra, IL-4, and IFN- $\gamma$ and a significant increase in the levels of IL-10. ${ }^{21}$ Another study on drug-naïve first-episode psychosis has indicated that the levels of IL-6, IL-10, TNF- $\alpha$, and IL-4 decreased significantly after risperidone treatment. ${ }^{22}$ However, a threemonth follow-up study of patients with SZ showed no significant differences in the levels of IL-2, IL-4, and IFN- $\gamma$ after risperidone was prescribed. ${ }^{23}$ Specifically, we analyzed the correlation between the changes in severity on the BPRS-E and cytokine levels. The changes in psychotic severity were significantly associated with the baseline IL-6 levels and changes in the levels of IFN- $\gamma$. Moreover, baseline severity correlated with the changes in the levels of IFN- $\gamma$ and IL-2. A previous study reported similar results: the baseline psychotic severity correlated with the baseline levels of IL- $6 .{ }^{24}$ Future studies with well-controlled participants may help to clarify the detailed mechanisms.

Another main result was that the levels of NCAM-1/CD56 were significantly decreased in the patients with SZ who were treated with SGAs but not FGAs. However, the differences in the levels of NCAM-1/CD56 were not significant regardless of the type of antipsychotic. NCAM-1, which is also known as CD56, is a member of the immunoglobulin superfamily, which is often considered a marker of neural lineage commitment and which is associated with natural killer cells. ${ }^{25}$ Previous studies have investigated the relationship between SZ and immunological alterations in lymphocytes, and no significant associations were found for NCAM-1/CD56. ${ }^{26,27} \mathrm{~A}$ meta-analysis ${ }^{28}$ has reported that the absolute levels of NCAM1/CD56 were significantly increased following antipsychotic treatment and that the percentages of CD4 and NCAM-1/ CD56 lymphocytes were significantly increased in acutely relapsed inpatients compared to controls. Therefore, future research will be necessary to clarify the immunological alterations of CD56 in SZ.

Several investigations have suggested a connection between SZ and changes in growth factors and/or neurotrophic factors, including BDNF, IGF-1, and IGFBP. ${ }^{29,30}$ With regard to IGFs, including IGFBP-1, IGFBP-2, and IGFBP-3, we did not find any significant differences in the levels of IGFBP after treatment. A 20 -week clinical trial ${ }^{31}$ of treatment with a program of resistance or concurrent exercise did not show significant changes in IGF-1 and IGFBP-3 levels in patients with SZ after treatment, which is similar to the results of the present study. However, our results showed significant correlations between the changes in severity and the baseline levels of IGFBP3, which suggests a potential treatment for future trials. We found no significant differences in the levels of GDNF and NGF after treatment. Compared with healthy subjects, patients with SZ exhibit decreased levels of NGF, ${ }^{32}$ and a random-effects metaanalysis showed similar results. ${ }^{33}$ Furthermore, GDNF levels have been shown to increase along with a reduction of psychotic symptoms in patients with SZ after antipsychotic treatment, ${ }^{10}$ but another study demonstrated that the serum levels of GDNF did not differ between patients with SZ and healthy controls. ${ }^{11}$ A cross-sectional observational study did not find significant differences in the levels of GDNF and NGF between responders and nonresponders treated with clozapine. ${ }^{34} \mathrm{Al}$ though no significant changes in BDNF levels after treatment were found, our results demonstrated that the baseline levels and changes in psychotic severity were significantly associated with changes in BDNF. Patients with SZ exhibit decreased levels of BDNF compared to healthy controls. ${ }^{32}$ Another prospective study did not find changes in BDNF levels in patients' serum, even though the psychosis severity significantly improved after six weeks of antipsychotic treatment. ${ }^{35}$ To summarize, the results of the present study suggests that BDNF, GDNF, and NGF may play a role in the progression of SZ, and additional studies are needed to help clarify this suggestion.

We reported the results of the comparisons of multiple groups of cytokines and assessments of psychotic severity. Thus, our results will be helpful for understanding the changes in the immunological, inflammatory, and neurodevelopment mechanisms that have been observed in patients suffering from chronic SZ. Furthermore, our eight-week study period extended across the acute and maintenance stages, which was a strength of the study. However, the following limitations of the study should be considered. First, the lack of a healthy control group and drug-naïve control group was a problem because placebo effects and other confounding factors might have affected our results. Second, because this was a naturalistic study, the antipsychotic agents were adjusted flexibly and therefore not well controlled. Finally, the relatively small number of patients enrolled in the study limits the generalizability of the results.

In conclusion, in this study, we found that the levels of IL1-ra were significantly decreased after eight weeks of antipsychotic treatment and that levels of NCAM-1/CD56 were significantly decreased in patients with SZ who received SGAs but not FGAs. Further studies are needed to confirm our results. 


\section{Acknowledgments}

We are grateful to our patients and their parents who voluntarily participated in our study and gave valuable information. This work was supported by grants VGHKS13-CT5-08 from Kaohsiung Veterans General Hospital, Taiwan.

\section{REFERENCES}

1. McGrath J, Saha S, Chant D, Welham J. Schizophrenia: a concise overview of incidence, prevalence, and mortality. Epidemiol Rev 2008;30: 67-76.

2. van Os J, Kapur S. Schizophrenia. Lancet 2009;374:635-645.

3. Millier A, Schmidt U, Angermeyer MC, Chauhan D, Murthy V, Toumi $\mathrm{M}$, et al. Humanistic burden in schizophrenia: a literature review. J Psychiatr Res 2014;54:85-93.

4. Howes $\mathrm{O}$, McCutcheon R, Stone J. Glutamate and dopamine in schizophrenia: an update for the 21st century. J Psychopharmacol 2015;29:97115.

5. Potvin S, Stip E, Sepehry AA, Gendron A, Bah R, Kouassi E. Inflammatory cytokine alterations in schizophrenia: a systematic quantitative review. Biol Psychiatry 2008;63:801-808.

6. Upthegrove R, Manzanares-Teson N, Barnes NM. Cytokine function in medication-naive first episode psychosis: a systematic review and meta-analysis. Schizophr Res 2014;155:101-108.

7. Nurjono M, Lee J, Chong SA. A Review of Brain-derived Neurotrophic Factor as a Candidate Biomarker in Schizophrenia. Clin Psychopharmacol Neurosci 2012;10:61-70.

8. Rizos EN, Papadopoulou A, Laskos E, Michalopoulou PG, Kastania A, Vasilopoulos D, et al. Reduced serum BDNF levels in patients with chronic schizophrenic disorder in relapse, who were treated with typical or atypical antipsychotics. World J Biol Psychiatry 2010;11:251-255.

9. Pillai A, Schooler NR, Peter D, Looney SW, Goff DC, Kopelowicz A, et al. Predicting relapse in schizophrenia: Is BDNF a plausible biological marker? Schizophr Res 2018;193:263-268.

10. Xiao W, Ye F, Ma L, Tang X, Li J, Dong H, et al. Atypical antipsychotic treatment increases glial cell line-derived neurotrophic factor serum levels in drug-free schizophrenic patients along with improvement of psychotic symptoms and therapeutic effects. Psychiatry Res 2016;246: 617-622.

11. Niitsu T, Shirayama Y, Matsuzawa D, Shimizu E, Hashimoto K, Iyo M. Association between serum levels of glial cell-line derived neurotrophic factor and attention deficits in schizophrenia. Neurosci Lett 2014; 575:37-41.

12. Martinez-Cengotitabengoa M, MacDowell KS, Alberich S, Diaz FJ, Garcia-Bueno B, Rodriguez-Jimenez R, et al. BDNF and NGF signalling in early phases of psychosis: relationship with inflammation and response to antipsychotics after 1 year. Schizophr Bull 2016;42:142-151.

13. Petrikis P, Boumba VA, Tzallas AT, Voulgari PV, Archimandriti DT, Skapinakis P, et al. Elevated levels of Insulin-like Growth Factor-1 (IGF1) in drug-naive patients with psychosis. Psychiatry Res 2016;246:348352 .

14. American Psychiatric Association. Diagnostic and Statistical Manual of Mental Disorders (5th Ed). Arlington, VA: American Psychiatric Publishing; 2013.

15. Overall JE, Gorham DR. The brief psychiaric rating scale. Psychol Rep 1962;10:799-812.

16. Ventura J, Lukoff D, Nuechterlein KH, Liberman RP, Green M, Shaner A. Appendix 1: Brief Psychiatric Rating Scale (BPRS) expanded version (4.0) scales, anchor points and administration manual. Int J Method Psychiatr Res 1993;3:227-243.
17. Ruggeri M, Koeter M, Schene A, Bonetto C, Vazquez-Barquero JL, Becker T, et al. Factor solution of the BPRS-expanded version in schizophrenic outpatients living in five European countries. Schizophr Res 2005;75:107-117.

18. de Witte L, Tomasik J, Schwarz E, Guest PC, Rahmoune H, Kahn RS, et al. Cytokine alterations in first-episode schizophrenia patients before and after antipsychotic treatment. Schizophr Res 2014;154:23-29.

19. Dinarello CA. The many worlds of reducing interleukin-1. Arthritis Rheum 2005;52:1960-1967.

20. Petersen AM, Pedersen BK. The anti-inflammatory effect of exercise. J Appl Physiol (1985) 2005;98:1154-1162.

21. Sobis J, Rykaczewska-Czerwinska M, Swietochowska E, Gorczyca P. Therapeutic effect of aripiprazole in chronic schizophrenia is accompanied by anti-inflammatory activity. Pharmacol Rep 2015;67:353-359.

22. Noto C, Ota VK, Gouvea ES, Rizzo LB, Spindola LM, Honda PH, et al. Effects of risperidone on cytokine profile in drug-naive first-episode psychosis. Int J Neuropsychopharmacol 2014;18.

23. Cazzullo CL, Sacchetti E, Galluzzo A, Panariello A, Adorni A, Pegoraro $\mathrm{M}$, et al. Cytokine profiles in schizophrenic patients treated with risperidone: a 3-month follow-up study. Prog Neuropsychopharmacol Biol Psychiatry 2002;26:33-39.

24. Pae CU, Yoon CH, Kim TS, Kim JJ, Park SH, Lee CU, et al. Antipsychotic treatment may alter T-helper (TH) 2 arm cytokines. Int Immunopharmacol 2006;6:666-671.

25. Cheng M, Chen Y, Xiao W, Sun R, Tian Z. NK cell-based immunotherapy for malignant diseases. Cell Mol Immunol 2013;10:230-252.

26. Rudolf S, Schlenke P, Broocks A, Peters M, Rothermundt M, Arolt V, et al. Search for atypical lymphocytes in schizophrenia. World J Biol Psychiatry 2004;5:33-37.

27. Steiner J, Jacobs R, Panteli B, Brauner M, Schiltz K, Bahn S, et al. Acute schizophrenia is accompanied by reduced $\mathrm{T}$ cell and increased $\mathrm{B}$ cell immunity. Eur Arch Psychiatry Clin Neurosci 2010;260:509-518.

28. Miller BJ, Gassama B, Sebastian D, Buckley P, Mellor A. Meta-analysis of lymphocytes in schizophrenia: clinical status and antipsychotic effects. Biol Psychiatry 2013;73:993-999.

29. Akanji AO, Ohaeri JU, Al-Shammri SA, Fatania HR. Associations of blood levels of insulin-like growth factor (IGF)-I, IGF-II and IGF binding protein (IGFBP)-3 in schizophrenic Arab subjects. Clin Chem Lab Med 2007;45:1229-1231.

30. Autry AE, Monteggia LM. Brain-derived neurotrophic factor and neuropsychiatric disorders. Pharmacol Rev 2012;64:238-258.

31. Silva BA, Cassilhas RC, Attux C, Cordeiro Q, Gadelha AL, Telles BA, et al. A 20-week program of resistance or concurrent exercise improves symptoms of schizophrenia: results of a blind, randomized controlled trial. Rev Bras Psiquiatr 2015;37:271-279.

32. Martinotti G, Di Iorio G, Marini S, Ricci V, De Berardis D, Di Giannantonio M. Nerve growth factor and brain-derived neurotrophic factor concentrations in schizophrenia: a review. J Biol Regul Homeost Agents 2012;26:347-356.

33. Qin XY, Wu HT, Cao C, Loh YP, Cheng Y. A meta-analysis of peripheral blood nerve growth factor levels in patients with schizophrenia. Mol Psychiatry 2017;22:1306-1312.

34. Krivoy A, Hochman E, Sendt KV, Hollander S, Vilner Y, Selakovic M, et al. Association between serum levels of glutamate and neurotrophic factors and response to clozapine treatment. Schizophr Res 2018;192: 226-231.

35. Pirildar S, Gonul AS, Taneli F, Akdeniz F. Low serum levels of brainderived neurotrophic factor in patients with schizophrenia do not elevate after antipsychotic treatment. Prog Neuropsychopharmacol Biol Psychiatry 2004;28:709-713. 